\title{
Surface Figuring of Large Carbon Fiber Reinforced Polymer Antenna Reflector with A Dual-robots Fabrication System
}

\author{
Qiang Xin ${ }^{1, *}$, Haitao $\mathrm{Liu}^{1}$, Jieli Wu${ }^{1}$, Lin Tang ${ }^{1}$, Dailu Wang ${ }^{1}$, and Yongjian Wan ${ }^{1}$ \\ ${ }^{1}$ Institute of Optics and Electronics, Chinese Academy of Sciences, NO.1 Guangdian Avenue Chengdu, China
}

\begin{abstract}
Carbon Fiber Reinforced Polymer (CFRP) has excellent physical and chemical properties which make it a promising material in making large space borne components, especially in making antenna reflectors and ultra-lightweight space mirrors. These components are usually in large scale to achieve the application requirements. In this research, a dual-robots fabrication system was in-house developed to meet the requirement for figuring a large off-axis parabolic CFRP antenna reflector with the size of $2.4 \mathrm{~m} \times 4.58 \mathrm{~m}$. To make sure that whole surface of the antenna reflector could be covered by the fabrication system, the surface was divided into six regions to accomplish the fabrication. In addition, a special designed tool was utilized to adapt to the curvature variation of the surface. The final surface form accuracies obtained for areas $\leqslant \phi$ $1750 \mathrm{~mm}$, $\leqslant$ \$ $2400 \mathrm{~mm}$ and the whole surface of the antenna reflector were $13.5 \mu \mathrm{m}$ RMS, $23.4 \mu \mathrm{m}$ RMS and $45.8 \mu \mathrm{m}$ RMS, respectively. Feasibility and surface figuring accuracy of the dual-robots system in fabricating large scale components were verified.
\end{abstract}

\section{Introduction}

With the development of space observation technology, sizes of the space telescope mirrors and satellite antenna reflectors are becoming bigger to achieve larger observation scope and high resolution. Weight of these space components are also getting larger. Therefore, scientists are facing the challenge of reducing weights of these space components to decrease the launch cost, and maintaining or promoting performances of the components at the meanwhile. Carbon Fiber Reinforced Polymer (CFRP) is a material with excellent physical chemical properties, such as high specific stiffness and low thermal expansion, which proposed an promising solution for making large space borne components with ultra-lightweight, especially in making satellite antenna reflector and ultra-lightweight space mirror[1,2].Size of these components are usually in scale of meters or even tens of meters which proposes great challenge in figuring their surfaces. In this research, a flexible dual-robots fabrication system was in-house developed to meet the requirements of figuring a large off-axis parabolic CFRP antenna reflector. The robots could work collaboratively in machining the reflector surface which could effectively raise the surface figuring efficiency and lower the time and economic costs.

\section{The CFRP antenna reflector}

Dimension of the off-axis parabolic CFRP antenna reflector to be figured is $2400 \mathrm{~mm} \times 4580 \mathrm{~mm}$ as shown in Fig. 1. Surface of the reflector has a radius of curvature of
$7992 \mathrm{~mm}$. The off-axis distance is 3898mm. Typical sandwich structure is utilized by the antenna whose skin and core are both made of CFRP. Study shows that this design has better performance for space borne antenna reflector in resisting the thermal deformation [1].

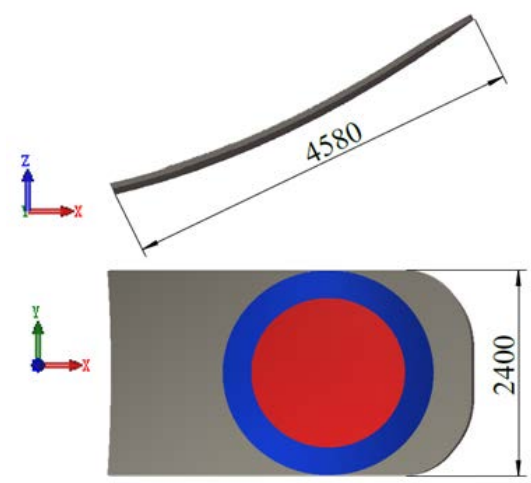

Fig. 1. CFRP antenna reflector

Root Mean Square error(RMS) of the surface form accuracy requirements are varied in different areas of the antenna reflector. Accuracies for areas $\leqslant \phi 1750 \mathrm{~mm}$ (as the red region shown in Fig.1), $\leqslant \phi 2400 \mathrm{~mm}$ (as the red and blue regions shown in Fig.1) and the whole surface are $15 \mu \mathrm{m}$ RMS, $30 \mu \mathrm{m}$ RMS and $90 \mu \mathrm{m}$ RMS, respectively.

\section{Dual-robots fabrication system and surface figuring method}

Arm length of the robot used in this research is $2.8 \mathrm{~m}$. However, the effective workspace of the robot is less than

\footnotetext{
* Corresponding author: xinqiang@ioe.ac.cn
} 
$2.8 \mathrm{~m}$ due to its position and posture when processing the antenna reflector. Moreover, the stiffness and the stability of the robot are decreased when robot working with its arm fully extended. Therefore, it is necessary to use two robots to fabricating the antenna cooperatively under its safe workspace with high stiffness and stability.

In this research, two robots were employed to achieve the surface figuring requirements. The robots were mounted on the rail guides besides the antenna as shown in Fig. 2. Each robot figured half surface of the antenna. They were positioned at different positions on the rail guides to fulfil the surface fabrication. Their positions were computed to assure that the whole surface of the antenna could be covered by the workspace of the robots. Fig. 2 presents the positions RA1, RA2 and RA3 for Robot No.1, and RB1, RB2 and RB3 for Robot No.2. Relations were then determined by accurately calibrating the antenna under coordinate of robots at different positions before the surface fabrication.

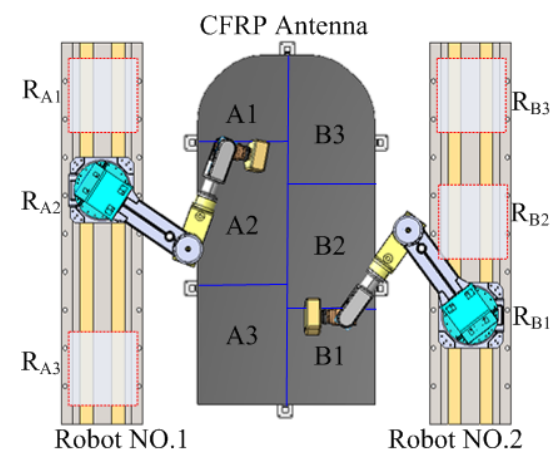

Fig. 2. Schematic of dual-robots fabrication system

A flexible lapping tool was employed to figure the surface of the antenna as shown in Fig. 3. The lapping tool could quickly adapt to the rapid change of surface curvature of the component. The tool moved in a spinning and orbital motion as the traditional one used in computer controlled optical surfacing. A classical meander rasterscanning tool path was adopted in figuring the antenna surface to obtain the required surface form accuracy. In order to lower the traces being produced in between the adjacent machining regions, overlap of the lapping tool in boundaries of the adjacent regions was deliberately considered in planning the surface figuring trajectory.

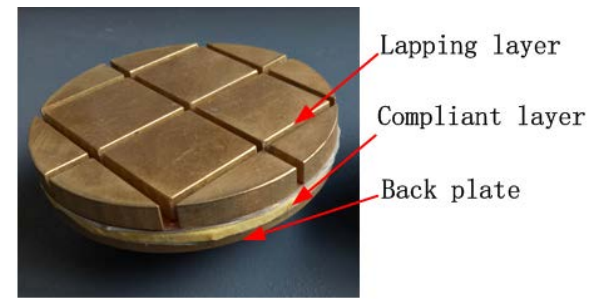

Fig. 3. Flexible lapping tool used in surface figuring

Because the surface form accuracy requirements were higher at the central areas ( $\leqslant \phi 1750 \mathrm{~mm}$ and $\leqslant \phi 2400$ ) than the rest one. These areas were extracted and handled specially to reach their accuracy targets.

\section{Results and discussion}

Surface form accuracy of the fabricated surface was measured by Radian laser tracker produced by Automatic Precision Inc. Before figuring, the surface form accuracies for areas $\leqslant \phi 1750 \mathrm{~mm}, \leqslant \phi 2400 \mathrm{~mm}$ and the whole surface were $42.3 \mu \mathrm{m}$ RMS, $51.7 \mu \mathrm{m}$ RMS and $56.9 \mu \mathrm{m}$ RMS, respectively. After figured by using the dual-robots system, the accuracies for areas $\leqslant \phi 1750 \mathrm{~mm}$, $\leqslant \phi 2400 \mathrm{~mm}$ and the whole surface were improved to $13.5 \mu \mathrm{m}$ RMS, $23.4 \mu \mathrm{m}$ RMS and $45.8 \mu \mathrm{m}$ RMS, respectively. Fig. 4 presents the surface form accuracies before and after the fabrication.

Because surface form accuracy at area $\leqslant \phi 1750 \mathrm{~mm}$ was higher, more material was removed to achieve its accuracy. Therefore, there is an obvious boundary between area $\leqslant \phi 1750 \mathrm{~mm}$ and areas $\leqslant \phi 2400 \mathrm{~mm}$ as shown in Fig. 4 b). To eliminate this phenomenon, an effective solution is to figure the central area firstly and reach its surface form accuracy, and then figure the rest surface with comparative material removal rate.

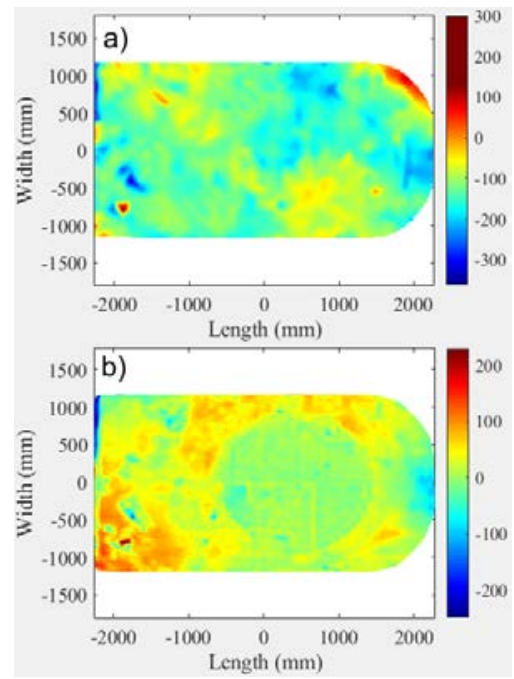

Fig. 4. Surface form accuracies of the antenna, a) before surface figuring and $b$ ) after surface figuring

\section{Summary}

A dual-robots fabrication system was in-house developed to meet the surface figuring requirements for a large offaxis parabolic CFRP antenna reflector with the size of $2.4 \mathrm{~m} \times 4.58 \mathrm{~m}$. The final surface form accuracies figured by using this fabrication system reached the accuracy targets. Flexibility and figuring accuracy of the fabrication system in processing large components were verified.

\section{References}

1. X. Zhou, X. Zhou, D. Du, X. Kong, G. Qin, Spacecraft Eng. 27, 83 (2018)

2. S. Utsunomiya, T. Kamiya, R. Shimizu, Proc. of SPIE, 8837, 88370P (2013) 